\title{
Commentary: Another step in the journey toward the "perfect" mitral valve repair
}

\author{
Vito Domenico Bruno, MD, PhD
}

\author{
From the Department of Translational Health Science, Bristol Medical School, University of Bristol, Bristol, \\ United Kingdom. \\ Disclosures: Author has nothing to disclose with regard to commercial support. \\ Received for publication May 18, 2019; accepted for publication May 22, 2019; available ahead of print June 27, \\ 2019. \\ Address for reprints: Vito Domenico Bruno, MD, PhD, Translational Health Sciences, Bristol Medical School, \\ University of Bristol, Research Floor Level 7, Bristol Royal Infirmary, Upper Maudlin St, BS2 8HW Bristol, \\ United Kingdom (E-mail: Vito.D.Bruno@bristol.ac.uk). \\ J Thorac Cardiovasc Surg 2020;159:1777-8 \\ 0022-5223/\$36.00 \\ Copyright (C) 2019 by The American Association for Thoracic Surgery \\ https://doi.org/10.1016/j.jtcvs.2019.05.028
}

How much do we know about the physiology of the mitral valve annulus? And what about the pathophysiology of mitral ring dehiscence? Can mitral ring technology improve even further? These questions instantly arose in my mind as soon as I finished reading the study of Ncho and colleagues ${ }^{1}$ published in the current issue of the Journal. With an excellent translational study that focused on the suture force dynamics involved in mitral valve ring annuloplasty, Ncho and colleagues ${ }^{1}$ have created a "heterogeneous" semiflexible ring and applied it in an advanced animal model of undersized annuloplasty. Their research team has an established experience with this type of model and has previously reported similar animal studies that investigated mitral ring suture forces. $^{2,3}$ In their previous reports, they found that the suture loading is influenced by ring type, ring annular size, and suture positions, and that dehiscence might not be related merely to isolated surgical errors. These findings are very important, and with this new study, Ncho and colleagues ${ }^{1}$ have gone even further by developing and creating a new semirigid 3-dimensionally printed mitral valve ring.

The information derived from this type of study is very helpful in clinical practice; undoubtedly the benefits of an animal model permit the researchers (and the surgeon) to gain information that otherwise would have been unavailable in a clinical study. In this sense, through a very sophisticated system of force transducers, Ncho and colleagues ${ }^{1}$ have identified the places where suture forces are higher (specifically the 1-o'clock and 11-o'clock positions) and have found that a semirigid ring provides a reduced suture force while preserving the remodeling ability of a fully rigid ring. Despite providing overall reduced suture forces, however, the semirigid ring showed no significant reduction on the anterior region and especially on the 1-o'clock and 11o'clock positions. This is a notable outcome, considering that in their previous works ${ }^{2,3}$ this group has emphasized

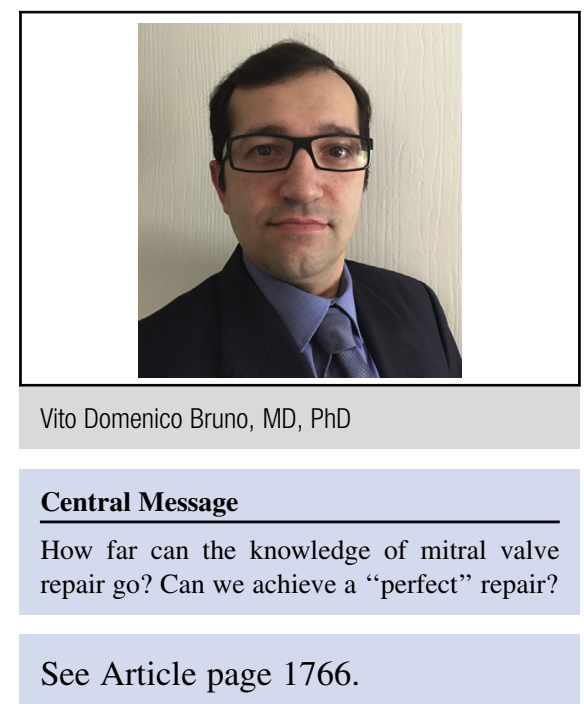

the higher loading of the anterior annulus relative to the posterior and commissural part, and it is particularly important considering that the interaction of ring type and suture region significantly affects the overall forces generated. This study has the limitations associated with most animal model studies, and it has been conducted only in a small sample of healthy animals. Moreover, the ovine model might not be the best for studying the mitral valve. Although mitral valve function in sheep is similar to that in humans, there are some differences in anatomic details. ${ }^{4}$ Translating these findings into human clinical practice would therefore require further studies and a larger number of in vitro and in vivo experiments. Surely, however, this article is captivating and, as an addition to the group's previous study, ${ }^{2}$ provides a good biomechanical understanding of the energies applied to the mitral valve annulus that can help surgeons in daily practice. Ncho and colleagues ${ }^{1}$ have also prepared a very nice video, in which they clearly describe the study protocol, the concept of the force transducer, and the main results of the study; it is certainly worthwhile to watch it.

Overall, this study is a very good example of a highquality, well-planned, and well-conducted translational study, and it provides a unique insight into the annuloplasty ring and its implication for the complex anatomic structure represented by the mitral valve annulus. In the modern era of mitral valve research, integration of knowledge among scientists, clinical researchers, and physicians is probably 
the most important step in the fascinating journey toward the "perfect" mitral valve repair.

\section{References}

1. Ncho BE, Pierce EL, Bloodworth CH IV, Imai A, Okamoto K, Saito Y, et al. Optimized mitral annuloplasty ring design reduces loading in the posterior annulus. $J$ Thorac Cardiovasc Surg. 2020;159:1766-74.e2.
2. Pierce EL, Bloodworth CH IV, Siefert AW, Easley TF, Takayama T, Kawamura T, et al. Mitral annuloplasty ring suture forces: impact of surgeon, ring, and use conditions. J Thorac Cardiovasc Surg. 2018;155:131-9.e3.

3. Pierce EL, Siefert AW, Paul DM, Wells SK, Bloodworth CH IV, Takebayashi S, et al. How local annular force and collagen density govern mitral annuloplasty ring dehiscence risk. Ann Thorac Surg. 2016;102:518-26.

4. Gorman JHI, Gupta KB, Streicher JT, Gorman RC, Jackson BM, Ratcliffe MB, et al. Dynamic three-dimensional imaging of the mitral valve and left ventricle by rapid sonomicrometry array localization. J Thorac Cardiovasc Surg. 1996;112:712-26. 